\title{
Exact three-colored quantum scars from geometric frustration
}

\author{
Kyungmin Lee $\odot,{ }^{1,2}$ Ronald Melendrez, ${ }^{1,2}$ Arijeet Pal, ${ }^{3}$ and Hitesh J. Changlani $\odot^{1,2}$ \\ ${ }^{1}$ Department of Physics, Florida State University, Tallahassee, Florida 32306, USA \\ ${ }^{2}$ National High Magnetic Field Laboratory, Tallahassee, Florida 32304, USA \\ ${ }^{3}$ Department of Physics and Astronomy, University College London, Gower Street, London WC1E 6BT, United Kingdom
}

(Received 14 March 2020; accepted 26 May 2020; published 12 June 2020)

\begin{abstract}
Nonequilibrium properties of quantum materials present many intriguing properties, among them athermal behavior, which violates the eigenstate thermalization hypothesis. Such behavior has primarily been observed in disordered systems. More recently, experimental and theoretical evidence for athermal eigenstates, known as "quantum scars," has emerged in nonintegrable disorder-free models in one dimension with constrained dynamics. In this Rapid Communication, we show the existence of quantum scar eigenstates and investigate their dynamical properties in many simple two-body Hamiltonians with "staggered" interactions, involving ferromagnetic and antiferromagnetic motifs, in arbitrary dimensions. These magnetic models include simple modifications of widely studied ones (e.g., the $X X Z$ model) on a variety of frustrated and unfrustrated lattices. We demonstrate our ideas by focusing on the two-dimensional frustrated spin-1/2 kagome antiferromagnet, which was previously shown to harbor a special exactly solvable point with "three-coloring" ground states in its phase diagram. For appropriately chosen initial product states-for example, those which correspond to any state of valid three-colors-we show the presence of robust quantum revivals, which survive the addition of anisotropic terms. We also suggest avenues for future experiments which may see this effect in real materials.
\end{abstract}

DOI: 10.1103/PhysRevB.101.241111

Introduction. How does an isolated quantum system "thermalize" given a particular set of initial conditions? This is one of the most basic questions of nonequilibrium dynamics of quantum matter in cold atom and condensed matter systems. The dynamics of an isolated quantum system at a macroscopic energy above the ground state is known to exhibit two universal behaviors: Either the system undergoes thermalization, or it is many-body localized (MBL) and fails to thermalize. The eigenstate thermalization hypothesis (ETH) [1-3] remarkably holds true for a wide variety of thermalizing systems, whereas it breaks down completely for MBL systems [4-8] or partially [9] in systems with conservation laws. Recent observations of long-lived periodic oscillations in one-dimensional Rydberg atom chains for a certain class of initial states [10] inspired the question of whether there are other alternatives to thermalization and many-body localization.

There are now various models in one [11-18] and higher dimensions $[19,20]$ where ETH is violated for a set of measure-zero highly excited eigenstates, known as manybody quantum scars, while the vast majority of eigenstates continue to satisfy ETH. It appears that scar eigenstates occur in the spectrum when the Hilbert space is fragmented due to kinetic constraints [21,22], thereby suppressing the relaxation of the initial state [23,24]. A major motivation for this Rapid Communication is to investigate the formation of ETH-violating excited states in frustrated magnetic systems potentially relevant for glassy dynamics in quantum magnets with degenerate energy landscapes [25-27]. The relevance of quantum dynamics at high energy to nonequilibrium effects in glassy spin systems remains a relatively unexplored question.

While not obviously directly related, ETH-violating athermal states appear instrumental in the observed quantum revivals [12,19]. This gives rise to a general prescription for observing scar states, which is to have a simple initial product state that has a large overlap with the athermal scar eigenstates. A constant energy spacing of the participating eigenstates guarantees the observations of a distinct revival timescale. The focus on "simple" states is crucial; while it is possible in theory to induce quantum oscillations between an arbitrary linear combination of a finite number of eigenstates, such a preparation may require control of nonlocal observables, which is experimentally challenging.

Given this prelude to quantum scars, we now elaborate the objective of this Rapid Communication, which is threefold. First, we present strategies that utilize geometric frustration for generating a large family of lattice Hamiltonians in arbitrary dimensions that have athermal states. Our prescription is general in nature, and shows that geometric frustration offers a route to constructing exponentially many scars in simple two(or few-) body quantum spin Hamiltonians. Second, we show that these idealized models show perfect revivals, and retain several aspects of the scar physics under perturbation (e.g., changing anisotropy). In addition, we also identify several unfrustrated models. And finally, we argue that a family of models may contain realistic candidates where revival effects will be observable on accessible timescales.

Before discussing a general recipe, we elucidate our key ideas with the help of a (quasi)exactly solvable point in the phase diagram of the nearest-neighbor $X X Z$ model on the spin$1 / 2$ kagome lattice,

$$
H_{X X Z}\left[J_{z}\right]=J \sum_{\langle i, j\rangle}\left(S_{i}^{x} S_{j}^{x}+S_{i}^{y} S_{j}^{y}\right)+J_{z} \sum_{\langle i, j\rangle} S_{i}^{z} S_{j}^{z},
$$



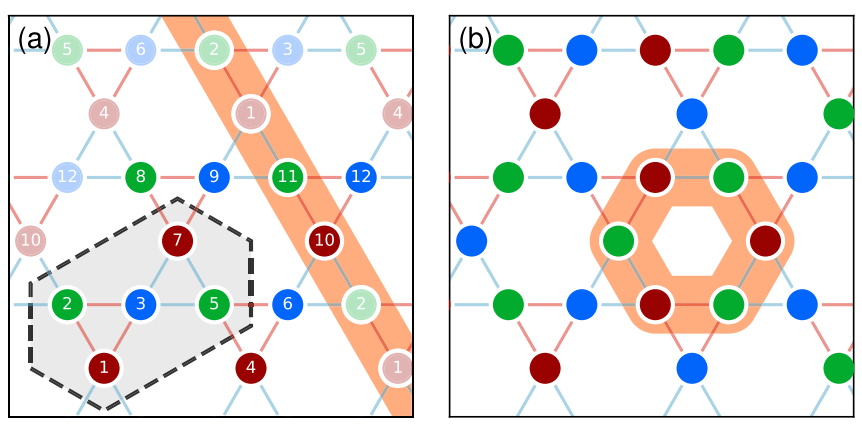

FIG. 1. Two representative three-colorings on the kagome lattice corresponding to two magnetically ordered configurations: (a) $q=0$ and (b) $\sqrt{3} \times \sqrt{3}$. The colors red, blue, and green represent the classical $120^{\circ}$ states or their quantum equivalents. The gray-shaded region in (a) indicates the subsystem used for the entanglement entropy result presented in Fig. 3. Two different red-green two-color loops are highlighted in orange.

where $S_{i}^{\alpha}$ are spin- $1 / 2$ operators on site $i$, and $\langle i, j\rangle$ refer to nearest-neighbor pairs. $J$ (set to 1 throughout) and $J_{z}$ are the $X Y$ and Ising couplings, respectively. We will denote the Hamiltonian $H_{X X Z}\left[J_{z}=-1 / 2\right]$ as $H_{X X Z 0}$, as in Ref. [28]. While the existence of a classical degeneracy and its quantum lifting in kagome magnets have been studied for a long time [28-32], Refs. [33,34] explicitly showed that, at this special point of $J_{z} / J=-1 / 2$, an exponential degeneracy exists in all $S_{z}$ sectors. The exact solutions apply to any lattice of triangular motifs with the Hamiltonian of the form $H=$ $\sum_{\triangle} H_{X X Z 0}(\triangle)$, where $H_{X X Z 0}(\triangle)$ is the XXZO Hamiltonian on a single triangular motif $\triangle$, as long as the vertices are consistently three-colorable, i.e., no two neighboring vertices have the same color. The proof relies on rewriting $H_{X X Z 0}$ in a frustration-free form as a sum of positive semidefinite projectors, and then showing that any product state of the following form is an exact ground state,

$$
|C\rangle=\prod_{s} \otimes\left|\gamma_{s}\right\rangle_{s}
$$

where $s$ is the site index and $\left|\gamma_{s}\right\rangle$ is one of $|r\rangle=(|\uparrow\rangle+$ $|\downarrow\rangle) / \sqrt{2}$ (red), $|g\rangle=(|\uparrow\rangle+\omega|\downarrow\rangle) / \sqrt{2}$ (green), or $|b\rangle=$ $\left(|\uparrow\rangle+\omega^{2}|\downarrow\rangle\right) / \sqrt{2}$ (blue), with $\omega=e^{i 2 \pi / 3}$. Examples of such colorings are shown in Fig. 1. (For details, refer to Refs. [33-35].)

Eigenstate entanglement structure for $H_{X X Z 0}$. We now discuss the properties of the three-coloring states $|C\rangle$ and their $S_{z}$ projections, with the intention of understanding why they are ETH violating. First, since the number of threecolorings on the kagome lattice scales exponentially with system size [36], there are exponentially many ground states at $J_{z} / J=-1 / 2$, each one of which is a product state. These states, however, are not orthogonal to each other, and break the U(1) symmetry of the $X X Z$ Hamiltonian. Projection to a particular $S_{z}$ sector restores the U(1) symmetry. The resulting state is still an eigenstate [33,34], and is weakly entangled: While the unprojected coloring state is a product state with zero entanglement, $S_{z}$ projection introduces entanglement that follows $S \sim \log V$ subvolume law [37]. (a)

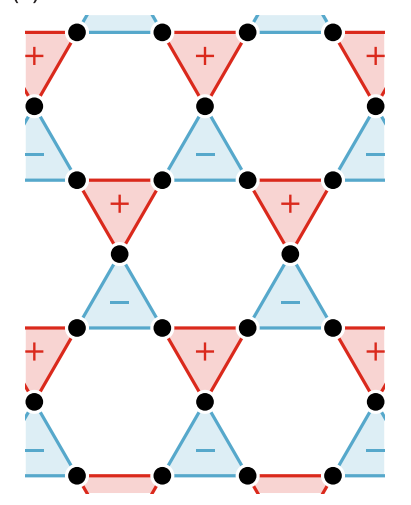

(b)

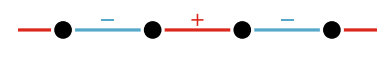

(c)

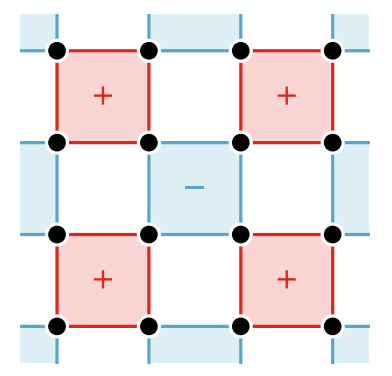

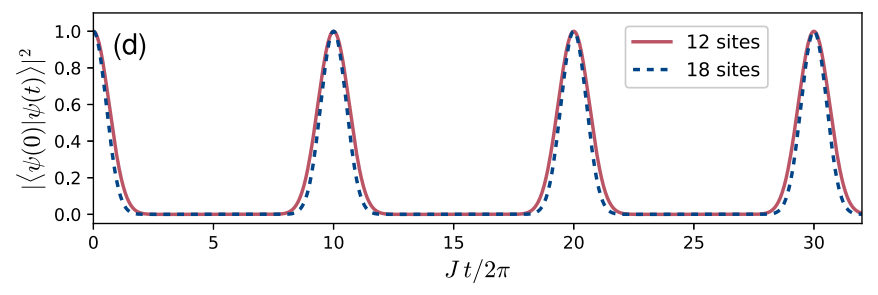

FIG. 2. (a)-(c) Lattices with alternating signs of interactions, i.e., "staggered" motifs: (a) kagome lattice, (b) one-dimensional chain, and (c) square lattice. The precise meaning of "+" and "-" is model dependent, and is explained in the text. (d) Perfect revival seen in the time evolution of $|\langle\psi(0) \mid \psi(t)\rangle|^{2}$ at $J_{z} / J=-1 / 2$ and $h / J=0.1$, with a $q=0$ three-coloring state as the initial state.

Importantly, despite existing at the same energy density, each three-coloring state has distinct local properties from most other three-coloring states. Consider, for example, the two three-colorings shown in the Fig. 1, the so-called $q=0$ and $\sqrt{3} \times \sqrt{3}$ coloring states. In either state one can identify "two-color" loops (examples highlighted in orange in Fig. 1). A new coloring state can be generated by color inverting a two-color loop (e.g., red $\leftrightarrow$ green). These effective tunnelings "connect" three-colorings to one another, yet, $q=0$ and $\sqrt{3} \times \sqrt{3}$ are not connected to each other via any local or topological move [38]. More generally, the three-coloring subspace fragments into topological and Kempe sectors [39]. The three-coloring manifold is a degenerate soup of quantum many-body states, magnetically ordered or disordered, all at exactly the same energy but arising from very different origins.

At face value, this observation might seem a quirk of lowenergy physics; after all, low-energy states are expected to be outside the realm of validity of ETH [40]. To show that this is not the case, and with the objective of making these states relevant at infinite temperature (i.e., the middle of the manybody spectrum), we modify the Hamiltonian such that all the "up" triangles have one sign of interaction, and all the "down" triangles have exactly the opposite sign, i.e.,

$$
H=\sum_{\nabla} H_{X X Z 0}(\nabla)-\sum_{\triangle} H_{X X Z 0}(\triangle) .
$$

A schematic for the kagome lattice is shown in Fig. 2(a), with "+" and "-" indicating the signs of the participating Hamiltonian pieces. This "staggered" construction destroys the 

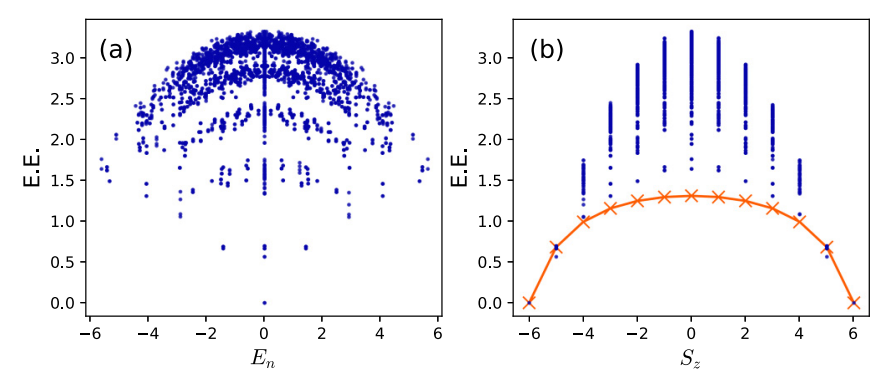

FIG. 3. Entanglement entropy of every eigenstate of the Hamiltonian in Eq. (3). The orange curve on the right panel indicates the EE of the $q=0$ state projected to each $S_{z}$ sector, which is expected to follow $\log V$ subvolume-law scaling.

positive semidefinite property of the Hamiltonian. Nevertheless, the three-coloring states (both projected and unprojected) still remain exact eigenstates, just not the lowest-energy ones [20]; they appear as zero-energy states in the many-body spectrum, which has a manifest $E \leftrightarrow-E$ symmetry by construction [see Supplemental Material (SM) [41]].

Our proposed prescription for constructing scar states with athermal dynamics is agnostic to whether or not the resultant Hamiltonian from the staggered construction is exactly integrable. Moreover, any arrangement of "+" and "-" which occurs in equal numbers will guarantee exact zero modes. In fact, even if the numbers of the "+" and "-" motifs are not exactly the same, or their interaction strengths are different, the coloring states remain eigenstates that lie in the interior of the many-body spectrum.

We explicitly check these analytic assertions with the help of numerical full diagonalizations. In Fig. 3(a), we show the entanglement entropy (EE) of every eigenstate computed on a 12-site system shown in Fig. 1(a) with periodic boundary conditions, as a function of the eigenstate energy $E_{n}$. Since there are exact degeneracies in the spectrum (especially the exponentially large degeneracy at $E=0$ ), the EE is not well defined for those states. Nevertheless, even if one focuses on nondegenerate states (for which the EE is reproducible), the $\mathrm{EE}$ is not a single-valued function of the energy as one would expect if ETH were to hold. While the model was originally designed to have ETH violation at $E=0$, we find that many more eigenstates at other energies also violate ETH.

To clarify the nature of the projected coloring states at $E=0$, we pick a representative three-coloring, here a translationally invariant $q=0$ state shown in Fig. 1(a). The EE of this state projected to every $S_{z}$ sector, shown in Fig. 3(b), follows $\log V$ subvolume law, and is lower than a majority of the eigenstates in the same sector [42].

Perfect quantum revivals in $H_{X X Z 0}$ from splitting degeneracy. Analogous to the quasidegenerate Anderson tower of states that appear in the low-energy spectra of unfrustrated magnets [43-48], for each three-coloring state, all its $S_{z}$ projections also form a tower. They are related by $\mathrm{U}(1)$ symmetry unlike the full (physical) $\mathrm{SU}(2)$ of the Heisenberg case. Importantly, the degeneracy in zero field is exact on any three-colorable lattice, which is split on adding a Zeeman term $H_{\text {Zeeman }}=-h \sum_{i} S_{i}^{z}$. The projected coloring states remain exact eigenstates, and only their energy changes; the
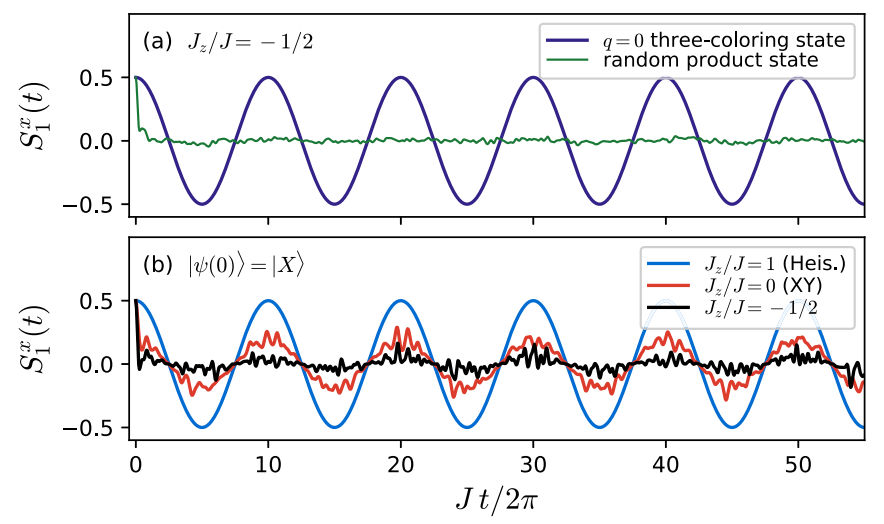

FIG. 4. $S_{1}^{x}$ vs time for $h / J=0.1$ in the 12-site system, (a) for two initial states $q=0$ three-coloring state (purple) and a random product state (green), both having $|r\rangle$ on site $1\left[S_{1}^{x}(t=0)=+1 / 2\right]$, at $J_{z} / J=-1 / 2$, and (b) for three values of anisotropy of the Hamiltonian, with the fully polarized $|X\rangle$ as the initial state.

degeneracy within every $S_{z}$ sector arising from different coloring configurations remains.

The unprojected three-coloring product state can be decomposed into $S_{z}$ sectors,

$$
|C\rangle=\sum_{S_{z}} P_{S_{z}}|C\rangle \equiv \sum_{S_{z}} \mathcal{N}_{S_{z}}\left|E_{S z}\right\rangle,
$$

where $\mathcal{N}_{S_{z}}$ is a sector-specific normalization factor and $\left|E_{S_{z}}\right\rangle$ is the normalized projected three-coloring eigenstate with energy $-h S_{z}$. Starting with the initial state $|\psi(t=0)\rangle=|C\rangle$, the Loschmidt echo thus gives

$$
\langle\psi(0) \mid \psi(t)\rangle \equiv \sum_{S_{z}} e^{-i t h S_{z}}\left|\mathcal{N}_{S_{z}}\right|^{2} .
$$

A characteristic timescale emerges from this expression which is $\tau=2 \pi / h$.

Numerical results shown in Fig. 2(d) confirm our analytic findings for the Loschmidt echo. Starting from a $q=0$ state, we observe that the echo shows perfect revivals which repeat with time period $\tau=2 \pi / h$. The profiles for both 12 and 18 sites are shown, and are consistent with the expectation that it gets sharper with increasing size. In contrast, if one starts with a random product state at the same energy density as the coherent state (i.e., $\langle H\rangle=0$ ) the memory of the initial state is rapidly lost (see SM [41] for further details).

These observations are further supported by dynamics of observables. We find perfect revivals in the time evolution of $S_{1}^{x}$, the $x$ component of the spin at site 1 , for a $q=0$ state [see Fig. 4(a)]; a random product state with $\left\langle S_{1}^{x}\right\rangle=+1 / 2$, on the other hand, shows a rapid decay.

Away from the special point. We now ask what happens away from the exactly solvable point $J_{z} / J=-1 / 2$ where the three-coloring states are no longer eigenstates of the Hamiltonian $H_{X X Z}$, and thus the quantum revival is not expected to be perfect. Will the same coherent state eventually thermalize? Figure 5 addresses this question. Indeed, we find that the revival is not perfect at $J_{z} / J=-0.4$. (Other anisotropies are discussed in SM [41].) What is surprising, however, is that the fidelity at long times saturates to a nonzero value (at least for 

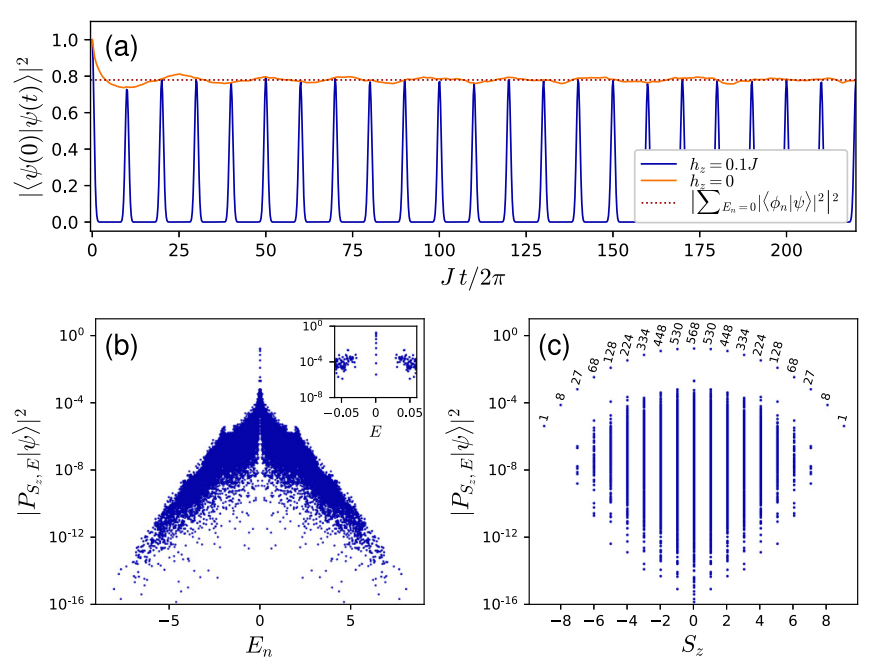

FIG. 5. Results at $J_{z} / J=-0.4$, away from the exactly solvable point. (a) Fidelity vs time, with $h=0.1 J$ (blue) and $h=0$ (orange) in an 18 site system. The red dotted line marks the overlap between the initial state and the zero-energy manifold (see SM [41]). (b), (c) Distribution of overlap with the $q=0$ state as a function (b) of $E_{n}$, and (c) of $S_{z}$. The numbers in (c) indicate the degeneracies.

the largest size we simulated) rather than slowly decaying to zero. This suggests the existence of scar states even away from the exactly solvable point; the fidelity saturates to the overlap between the participating states (i.e., the scar manifold) and the initial state (i.e., the $q=0$ state).

Figures 5(b) and 5(c) show the distribution of the overlap between the eigenstate manifolds and the $q=0$ state, respectively, as functions of $E_{n}$ and $S_{z}$. As shown in Fig. 5(b), and more clearly in its inset, a group of zero-energy states exist, with large overlaps with the $q=0$ state. These states comprise the scar manifold at $J_{z} / J=-0.4$. Furthermore, when plotted as a function of $S_{z}$, the scar manifold is clearly separated from all the other states [see Fig. 5(c)]. This manifold remains highly degenerate even within each $S_{z}$ sector, as indicated by the numbers in Fig. 5(c). (See SM [41] for details of calculating the overlap.)

Generalization to other models. Based on the analyses of the kagome model, we surmise that the key ingredients for perfect revivals are the following: (a) Generate a perfect degeneracy in the spectrum. One mechanism to do this is to have states in different $S_{z}$ sectors to be all degenerate. (b) Split this degeneracy with a field (here, the Zeeman term). (c) Prepare the system in a simple initial state, preferably a product state. These ingredients resonate with Schecter and Iadecola's [19] observation of scars in spin-1 $X Y$ magnets on hypercubic lattices: They found an expression similar to Eq. (5), but in a very different model from ours. This raises a natural question: Is there a more general way to generate lattice Hamiltonians and initial conditions which satisfy criteria (a)-(c)?

Here, we offer two possible routes. The first route is to leverage properties of highly frustrated latticessuch as the kagome, hyperkagome, or pyrochlore-to engineer a Hamiltonian that makes any of its valid "colorings" an exact eigenstate $[33,49,50]$. This recipe is equivalent to finding the operators which annihilate the coloring states. (See SM [41] for further discussion and how a representative calculation was done with the DIRACQ package [51].) The phase space of such Hamiltonians is large [33], although not all of them consist solely of two-spin interactions.

The second route is to focus on models with isotropic two-spin Heisenberg interactions on lattices, with or without frustration, and introduce "staggered" interactions [examples are shown in Figs. 2(a)-2(c)]-more precisely, a Hamiltonian of the form $H=\sum_{+ \text {motifs }} H^{+}\left[J_{z}=J\right]-\sum_{- \text {motifs }} H^{-}\left[J_{z}=\right.$ $J$ ]. In all such models, $S U(2)$ symmetry guarantees that the fully polarized (i.e., ferromagnetic) state $|S, S\rangle \equiv|\uparrow \uparrow \uparrow \cdots\rangle$ and all other members of the multiplet $\left|S, S_{z}\right\rangle$ are eigenstates with exactly zero energy. Once again, applying a magnetic field splits the degeneracy of this multiplet, while retaining the eigenstate structure. For example, an initial state with all spins pointing in the $x$ direction $|X\rangle=\bigotimes(|\uparrow\rangle+|\downarrow\rangle) / \sqrt{2}$ under magnetic field $\mathbf{B}=h \hat{z}$ leads to an expression identical to Eq. (5) for the Loschmidt echo. Furthermore, tuning away from the Heisenberg point also leads to imperfect revivals, as can be observed in the local spin measurement [see Fig. 4(b)].

Conclusions and future prospects. Quantum revivals are well studied in the context of Rabi oscillations of two-level systems (e.g., single spin in a magnetic field). The crucial difference in the case of scars is that it is a macroscopic spin that is precessing, not allowing the system to thermalize. This effect arises from the special choice of initial conditions and the nature of the many-body spectrum in our proposed models. Among our proposed models, we believe that the "second route" of Heisenberg interactions with staggered motifs may be a realistic possibility. A possible experimental protocol, which parallels that used in NMR experiments, is to place the candidate material in a static magnetic field in one direction (e.g., in the $z$ direction), and then to apply a much larger magnetic field transverse to it to polarize the starting state of spins in that direction (e.g., in the $x$ direction) for a time much shorter than the time period of the scar oscillations $\tau=2 \pi \hbar / g \mu_{B} h$, where the $g$ factor depends on the effective magnetic moment. Assuming that $g$ in the spin (or pseudospin) Hamiltonians can vary on a scale of 1-10 [52,53], and that fields of 0.001-10 $\mathrm{T}$ are applied, timescales are of orders $10^{-12}-10^{-7}$ s. Note that this time period of oscillation is completely independent of the magnetic coupling strength $J$, and thus we believe that this effect could be observable in a wide class of materials (were they to exist) with staggered interactions.

Acknowledgments. We thank O. Vafek, V. Dobrosavljevic, S. Bramwell, G. Baskaran, D. McMorrow, C. Laumann, and S. Pujari for particularly inspiring discussions. H.J.C. thanks B. Clark, S. Pujari, C. Chung, D. Kochkov, K. Kumar, E. Fradkin, and (the late) C. L. Henley for earlier collaborations on related topics in frustrated magnetism. He would also like to thank the hospitality of Amit Ghosal and IISER Kolkata, where part of this work was completed. K.L., R.M., and H.J.C. acknowledge support from Florida State University and the National High Magnetic Field Laboratory. The National High Magnetic Field Laboratory is supported by the National Science Foundation through Grant No. DMR-1644779 and by the state of Florida. A.P. was funded by the European Research Council (ERC) under the European Union's 
Horizon 2020 research and innovation programme (Grant Agreement No. 853368). We also thank the Research Com- puting Cluster (RCC) at Florida State University for computing resources.
[1] J. M. Deutsch, Quantum statistical mechanics in a closed system, Phys. Rev. A 43, 2046 (1991).

[2] M. Srednicki, Chaos and quantum thermalization, Phys. Rev. E 50, 888 (1994).

[3] M. Rigol, V. Dunjko, and M. Olshanii, Thermalization and its mechanism for generic isolated quantum systems, Nature (London) 452, 854 (2008).

[4] D. M. Basko, I. L. Aleiner, and B. L. Altshuler, Metal-insulator transition in a weakly interacting many-electron system with localized single-particle states, Ann. Phys. (NY) 321, 1126 (2006).

[5] A. Pal and D. A. Huse, Many-body localization phase transition, Phys. Rev. B 82, 174411 (2010).

[6] V. Oganesyan and D. A. Huse, Localization of interacting fermions at high temperature, Phys. Rev. B 75, 155111 (2007).

[7] R. Nandkishore and D. A. Huse, Many-body localization and thermalization in quantum statistical mechanics, Annu. Rev. Condens. Matter Phys. 6, 15 (2015).

[8] D. A. Abanin, E. Altman, I. Bloch, and M. Serbyn, Colloquium: Many-body localization, thermalization, and entanglement, Rev. Mod. Phys. 91, 021001 (2019).

[9] O. Vafek, N. Regnault, and B. A. Bernevig, Entanglement of exact excited eigenstates of the Hubbard model in arbitrary dimension, SciPost Phys. 3, 043 (2017).

[10] H. Bernien, S. Schwartz, A. Keesling, H. Levine, A. Omran, H. Pichler, S. Choi, A. S. Zibrov, M. Endres, M. Greiner, V. Vuletic, and M. D. Lukin, Probing many-body dynamics on a 51-atom quantum simulator, Nature (London) 551, 579 (2017).

[11] N. Shiraishi and T. Mori, Systematic Construction of Counterexamples to the Eigenstate Thermalization Hypothesis, Phys. Rev. Lett. 119, 030601 (2017).

[12] C. J. Turner, A. A. Michailidis, D. A. Abanin, M. Serbyn, and Z. Papic, Weak ergodicity breaking from quantum many-body scars, Nat. Phys. 14, 745 (2018).

[13] C. J. Turner, A. A. Michailidis, D. A. Abanin, M. Serbyn, and Z. Papić, Quantum scarred eigenstates in a Rydberg atom chain: Entanglement, breakdown of thermalization, and stability to perturbations, Phys. Rev. B 98, 155134 (2018).

[14] S. Moudgalya, S. Rachel, B. A. Bernevig, and N. Regnault, Exact excited states of nonintegrable models, Phys. Rev. B 98, 235155 (2018).

[15] S. Moudgalya, N. Regnault, and B. A. Bernevig, Entanglement of exact excited states of Affleck-Kennedy-Lieb-Tasaki models: Exact results, many-body scars, and violation of the strong eigenstate thermalization hypothesis, Phys. Rev. B 98, 235156 (2018).

[16] C.-J. Lin and O. I. Motrunich, Exact Quantum Many-Body Scar States in the Rydberg-Blockaded Atom Chain, Phys. Rev. Lett. 122, 173401 (2019).

[17] V. Khemani, C. R. Laumann, and A. Chandran, Signatures of integrability in the dynamics of Rydberg-blockaded chains, Phys. Rev. B 99, 161101(R) (2019).

[18] K. Bull, I. Martin, and Z. Papić, Systematic Construction of Scarred Many-Body Dynamics in 1D Lattice Models, Phys. Rev. Lett. 123, 030601 (2019).
[19] M. Schecter and T. Iadecola, Weak Ergodicity Breaking and Quantum Many-Body Scars in Spin-1 XY Magnets, Phys. Rev. Lett. 123, 147201 (2019).

[20] S. Ok, K. Choo, C. Mudry, C. Castelnovo, C. Chamon, and T. Neupert, Topological many-body scar states in dimensions one, two, and three, Phys. Rev. Research 1, 033144 (2019).

[21] P. Sala, T. Rakovszky, R. Verresen, M. Knap, and F. Pollmann, Ergodicity Breaking Arising from Hilbert Space Fragmentation in Dipole-Conserving Hamiltonians, Phys. Rev. X 10, 011047 (2020).

[22] V. Khemani, M. Hermele, and R. Nandkishore, Localization from Hilbert space shattering: From theory to physical realizations, Phys. Rev. B 101, 174204 (2020).

[23] S. Choi, C. J. Turner, H. Pichler, W. W. Ho, A. A. Michailidis, Z. Papić, M. Serbyn, M. D. Lukin, and D. A. Abanin, Emergent SU(2) Dynamics and Perfect Quantum Many-Body Scars, Phys. Rev. Lett. 122, 220603 (2019).

[24] C.-J. Lin, A. Chandran, and O. I. Motrunich, Slow thermalization of exact quantum many-body scar states under perturbations, arXiv:1910.07669.

[25] C. Chamon, Quantum Glassiness in Strongly Correlated Clean Systems: An Example of Topological Overprotection, Phys. Rev. Lett. 94, 040402 (2005).

[26] M. van Horssen, E. Levi, and J. P. Garrahan, Dynamics of many-body localization in a translation-invariant quantum glass model, Phys. Rev. B 92, 100305(R) (2015).

[27] Z. Lan, M. van Horssen, S. Powell, and J. P. Garrahan, Quantum Slow Relaxation and Metastability Due to Dynamical Constraints, Phys. Rev. Lett. 121, 040603 (2018).

[28] K. Essafi, O. Benton, and L. D. C. Jaubert, A kagome map of spin liquids from $X X Z$ to Dzyaloshinskii-Moriya ferromagnet, Nat. Commun. 7, 10297 (2016).

[29] A. B. Harris, C. Kallin, and A. J. Berlinsky, Possible Néel orderings of the kagomé antiferromagnet, Phys. Rev. B 45, 2899 (1992).

[30] J. T. Chalker, P. C. W. Holdsworth, and E. F. Shender, Hidden Order in A Frustrated System: Properties of the Heisenberg Kagomé Antiferromagnet, Phys. Rev. Lett. 68, 855 (1992).

[31] D. A. Huse and A. D. Rutenberg, Classical antiferromagnets on the kagomé lattice, Phys. Rev. B 45, 7536 (1992).

[32] C. L. Henley, Long-range order in the classical kagome antiferromagnet: Effective Hamiltonian approach, Phys. Rev. B 80, 180401(R) (2009).

[33] H. J. Changlani, D. Kochkov, K. Kumar, B. K. Clark, and E. Fradkin, Macroscopically Degenerate Exactly Solvable Point in the Spin-1/2 Kagome Quantum Antiferromagnet, Phys. Rev. Lett. 120, 117202 (2018).

[34] H. J. Changlani, S. Pujari, C.-M. Chung, and B. K. Clark, Resonating quantum three-coloring wave functions for the kagome quantum antiferromagnet, Phys. Rev. B 99, 104433 (2019).

[35] T. Momoi and M. Suzuki, Ground-state properties and phase diagram of the quantum $X X Z$ antiferromagnet on a triangular lattice, J. Phys. Soc. Jpn. 61, 3732 (1992).

[36] R. J. Baxter, Colorings of a hexagonal lattice, J. Math. Phys. 11, 784 (1970). 
[37] This holds true for any generic product state projected to a specific $S_{z}$ sector. See Supplemental Material for the proof of $S \sim \log V[41]$.

[38] C. Castelnovo, C. Chamon, C. Mudry, and P. Pujol, Quantum three-coloring dimer model and the disruptive effect of quantum glassiness on its line of critical points, Phys. Rev. B 72, 104405 (2005).

[39] O. Cépas and A. Ralko, Resonating color state and emergent chromodynamics in the kagome antiferromagnet, Phys. Rev. B 84, 020413(R) (2011).

[40] A. M. Läuchli, J. Sudan, and R. Moessner, $s=\frac{1}{2}$ kagome Heisenberg antiferromagnet revisited, Phys. Rev. B 100, 155142 (2019).

[41] See Supplemental Material at http://link.aps.org/supplemental/ 10.1103/PhysRevB.101.241111 for $E \leftrightarrow-E$ symmetry of the staggered Hamiltonian; overlap of a three-coloring state and a degenerate manifold; a prescription for generating a fourcoloring scar-bearing model; how the memory effect in the Loschmidt echo depends on the initial state for various anisotropies; stability of the scar manifold; and a proof of $S \sim \log V$ entanglement entropy of projected product states.

[42] The question of ETH for degenerate states is ill defined, since the entanglement entropy depends on the choice of linear combination. Despite this ambiguity, it is clear that the projected colorings do not have the entanglement of a typical high-energy (infinite temperature) state.

[43] P. W. Anderson, An approximate quantum theory of the antiferromagnetic ground state, Phys. Rev. 86, 694 (1952).

[44] B. Bernu, C. Lhuillier, and L. Pierre, Signature of Néel Order in Exact Spectra of Quantum Antiferromagnets on Finite Lattices, Phys. Rev. Lett. 69, 2590 (1992).
[45] C. Lhuillier, Frustrated quantum magnets, arXiv:condmat/0502464.

[46] H. Neuberger and T. Ziman, Finite-size effects in Heisenberg antiferromagnets, Phys. Rev. B 39, 2608 (1989).

[47] H. J. Changlani, S. Ghosh, C. L. Henley, and A. M. Läuchli, Heisenberg antiferromagnet on Cayley trees: Low-energy spectrum and even/odd site imbalance, Phys. Rev. B 87, 085107 (2013).

[48] H. J. Changlani, S. Ghosh, S. Pujari, and C. L. Henley, Emergent Spin Excitations in a Bethe Lattice at Percolation, Phys. Rev. Lett. 111, 157201 (2013).

[49] E. Chertkov and B. K. Clark, Computational Inverse Method for Constructing Spaces of Quantum Models from Wave Functions, Phys. Rev. X 8, 031029 (2018).

[50] Y. Wan and M. J. P. Gingras, Color ice states, weathervane modes, and order by disorder in the bilinear-biquadratic pyrochlore Heisenberg antiferromagnet, Phys. Rev. B 94, 174417 (2016).

[51] J. G. Wright and B. S. Shastry, DiracQ: A quantum many-body physics package, arXiv:1301.4494.

[52] A. Scheie, J. Kindervater, S. Zhang, H. J. Changlani, G. Sala, G. Ehlers, A. Heinemann, G. S. Tucker, S. M. Koohpayeh, and C. Broholm, Multiphase magnetism in $\mathrm{Yb}_{2} \mathrm{Ti}_{2} \mathrm{O}_{7}$, arXiv:1912.04913.

[53] K. W. Plumb, H. J. Changlani, A. Scheie, S. Zhang, J. W. Krizan, J. A. Rodriguez-Rivera, Y. Qiu, B. Winn, R. J. Cava, and C. L. Broholm, Continuum of quantum fluctuations in a three-dimensional $S=1$ Heisenberg magnet, Nat. Phys. 15, 54 (2018). 